\title{
Comprehensive stochastic sensitivities to resonance parameters
}

\author{
Pierre TAMAGNO ${ }^{1, *}$ and Elias VANDERMEERSCH ${ }^{1}$, \\ ${ }^{1}$ CEA/DER/SPRC/LEPh, CEA Cadarache, 13108 Saint-Paul-lez-Durance, France
}

\begin{abstract}
Integral experiments in reactors or critical configurations claim to have very small experimental and technological uncertainties. Therefore these latter can be considered valuable experimental information in nuclear data evaluation. Because in the evaluation process the information is carried by model parameters, to perform a rigorous feedback on a nuclear model parameters $p$ - for instance using a measured reactivity $\rho-$ sensitivities $S=\frac{\partial \rho / \rho}{\partial p / p}$ are needed. In usual integral feedbacks, sensitivity to multi-group cross sections are first obtained with deterministic code using perturbation theory. Then these multi-group cross section sensitivities are "convoluted" with parameter sensitivities in order to provide the sensitivity on nuclear model parameter. Recently stochastic approaches have been elaborated in order to obtain continuous cross-section sensitivities thus avoiding the multi-group discretization. In the present work we used the recent Iterated Fission Probability method of the TRIPOLI4 code [1] in order to obtain directly the sensitivity to nuclear physics parameters. We focus here on the sensitivity on resonance parameters and exemplified the method on the computation of sensitivities for ${ }^{239} \mathrm{Pu}$ and ${ }^{16} \mathrm{O}$ resonance parameters one the ICSBEP benchmark PST001. The underlying nuclear model describing resonant cross sections are based in the R-matrix formalism [2] that provides not only the interaction cross sections but also the angular distribution of the scattered neutrons i.e. differential cross sections. The method has thus been updated in order to compute parameter sensitives that include both contributions: cross section and angular distributions. This extension of the method was tested with exact perturbation of angular distribution and fission spectrum.
\end{abstract}

\section{Introduction}

In nuclear systems the steady-state neutron flux at given phase-sate $\epsilon=(\vec{r}, \hat{\Omega}, E)$ is driven by the Boltzmann equation

$$
\begin{aligned}
& \hat{\Omega} \cdot \vec{\nabla} \psi(\epsilon)+\sum_{i} N_{i}(\vec{r}) \sigma_{\mathrm{tot}, i}(E) \psi(\epsilon)= \\
& \sum_{i} \int \mathrm{d} \epsilon^{\prime} v_{\mathrm{scat}, i}\left(E^{\prime}\right) N_{i}\left(\vec{r}^{\prime}\right) \sigma_{\mathrm{scat}, i}\left(E^{\prime}\right) p_{\mathrm{scat}, i}\left(\epsilon^{\prime} \rightarrow \epsilon\right) \psi\left(\epsilon^{\prime}\right) \\
& +\frac{1}{k_{\mathrm{eff}}} \sum_{i} \int \mathrm{d} \epsilon^{\prime} v_{\mathrm{fiss}, i}\left(E^{\prime}\right) N_{i}\left(\vec{r}^{\prime}\right) \sigma_{\mathrm{fiss}, i}\left(E^{\prime}\right) p_{\mathrm{fiss}, i}\left(\epsilon^{\prime} \rightarrow \epsilon\right) \psi\left(\epsilon^{\prime}\right)
\end{aligned}
$$

where the generic $v_{\mathrm{scat}, i}$ can stands for $n, x n$ reactions. In the last term - corresponding to the fission production - the sum runs over the fissile isotopes only, giving: $p_{\text {fiss }, i}\left(\epsilon^{\prime} \rightarrow \epsilon\right)=\frac{\chi_{i}\left(E^{\prime} \in E\right)}{4 \pi} \delta_{\vec{r}, \vec{r}^{\prime}}$.

The $k_{\text {eff }}$ eigenvalue in Eq. 1 is a parameter of major importance as it mainly describes the tendency of the system to depart from criticality. With $k_{\text {eff }}>1$ the neutron population (and the generated power) increases whereas for $k_{\text {eff }}<1$ the neutron population vanishes. The accurate computation of such a parameter is of major importance for safety and criticality as it defines the safe range of operation, for instance where the evolution time-scale of neutron population is large enough to be controlled by mechanical devices.

\footnotetext{
*e-mail: pierre.tamagno@cea.fr
}

The current computational power allows to use stochastic codes for real scale nuclear systems, so that the major contributor to the uncertainty on the computed $k_{\text {eff }}$ comes now from nuclear data. The computation of $k_{\text {eff }}$ requires a large amount of nuclear data: reactions cross sections, neutron multiplicities and distributions for each isotope present in the system. It is therefore a tremendous task to propagate nuclear data uncertainties or to get parameter sensitivity using brute-force calculations.

In most cases nuclear data (cross section, etc.) are produced from the evaluation (fitting) of nuclear model parameters. One should actually focus on the propagation of uncertainties related to the nuclear model parameter values, not to produced nuclear data. However in evaluated nuclear data files (in ENDF6 format) no model parameter are provided with the exception of resonance parameters. Therefore in integral data assimilation the link with nuclear model parameters is often broken one eventually obtain adjusted libraries. Recent studies have attempted to restore the link between evaluated nuclear model parameters and integral parameters [3]. However while restoring the link, one should keep in mind that nuclear data stored in evaluated files are divided according to their nature (cross section, angular distribution, etc.) and that this "storing system" hides their common origin. Nuclear model codes used in the evaluation actually produce several types of nuclear data, for instance cross sections and angular distributions, based on the same set of parame- 
ters. A rigorous feedback from integral experiments to basic nuclear model parameters must keep this link. In this work we present how to compute accurately the sensitivity coefficient $S_{p}^{\rho}$ of the reactivity $\rho$ with respect of the nuclear model parameters $p$.

$$
S_{p}^{\rho}=\frac{\partial \rho}{\partial p / p}
$$

The presented application is limited to the case of resonance parameters but this approach can be generalized to other nuclear model parameters.

\section{Sensitivities on Nuclear Data}

An elegant perturbation theory [4] allows to compute the exact impact on $k_{\text {eff }}$ due to a change in the input data (nuclear data or concentrations). Instead of working with $k_{\text {eff }}$ we choose here to work with the reactivity $\rho=1-1 / k_{\text {eff }}$. With this formalism the perturbation $\Delta \rho$ due to a change $\Delta L$ or $\Delta P$ in the Boltzmann equation (written as $\frac{1}{k_{\mathrm{eff}}} P \psi-$ $L \psi=0)$ is given by

$$
\Delta \rho=\frac{\left\langle\psi_{2}^{\dagger}\left(\frac{1}{k_{\mathrm{eff}}} \Delta P-\Delta L\right) \psi_{1}\right\rangle_{\epsilon}}{\left\langle\psi_{2}^{\dagger} P_{1} \psi_{1}\right\rangle_{\epsilon}}
$$

where $\psi_{1}$ is the direct flux computed in the "reference" state and $\psi_{2}^{\dagger}$ is the adjoint flux computed in the "perturbed" state. This expression correspond to an exact perturbation. If one considers only small perturbations, the adjoint flux $\psi_{2}^{\dagger}$ can be replaced by $\psi_{1}^{\dagger}$, so that we obtained the first order perturbation

$$
\delta \rho=\frac{\left\langle\psi_{1}^{\dagger}\left(\frac{1}{k_{\mathrm{eff}}} \Delta P-\Delta L\right) \psi_{1}\right\rangle_{\epsilon}}{\left\langle\psi_{1}^{\dagger} P_{1} \psi_{1}\right\rangle_{\epsilon}}
$$

One can see that this formalism allows to compute any first-order perturbation with a single computation of $\psi_{1}, \psi_{1}^{\dagger}$ and $k_{\text {eff }}$. For a parameter $q$ (cross section or nuclear model parameter), the sensitivity coefficients can be expressed as

$$
S_{q}^{\rho}=\frac{\partial \rho}{\partial q} q \approx \frac{\delta \rho}{\delta q} q \approx \frac{q\left\langle\psi_{1}^{\dagger}\left(\frac{1}{k_{\mathrm{eff}}} \frac{\partial P}{\partial q}-\frac{\partial L}{\partial q}\right) \psi_{1}\right\rangle_{\epsilon}}{\left\langle\psi_{1}^{\dagger} P_{1} \psi_{1}\right\rangle_{\epsilon}}
$$

We can define the effective multiplicity $\tilde{v}_{i, r}(E)$ as

$$
\tilde{v}_{i, r}(E)= \begin{cases}0, & r=\mathrm{capt} \\ v_{i, r}(E) & r=\text { scat } \\ \frac{v_{i, r}(E)}{k_{\mathrm{eff}}} & r=\text { fiss }\end{cases}
$$

so that we can write the generic expression for the capture, scattering or fission cross section. The sensitivity coefficient $S_{\sigma_{r, i}(E)}^{\rho}$ to the reaction cross section $\sigma_{r, i}$ is thus given by

$$
\begin{gathered}
S_{\sigma_{r, i}(E)}^{\rho}=\frac{\sigma_{r, i}(E)}{\left\langle\psi_{1}^{\dagger} P_{1} \psi_{1}\right\rangle_{\epsilon}} \\
\times\left\langle\left[\tilde{v}_{r, i}(E)\left\langle p_{r, i}\left(\epsilon \rightarrow \epsilon^{\prime}\right) \psi_{1}^{\dagger}\left(\epsilon^{\prime}\right)\right\rangle_{\epsilon^{\prime}}-\psi_{1}^{\dagger}(\epsilon)\right] N_{i}(\vec{r}) \psi_{1}(\epsilon)\right\rangle_{\vec{r}, \hat{\Omega}}
\end{gathered}
$$

and for the related distribution

$$
S_{p_{r, i}\left(\epsilon \rightarrow \epsilon^{\prime}\right)}^{\rho}=\frac{p_{r, i}\left(\epsilon \rightarrow \epsilon^{\prime}\right) \psi_{1}^{\dagger}\left(\epsilon^{\prime}\right) \tilde{v}_{r, i}(E) \sigma_{r, i}(E) N_{i}(\vec{r}) \psi_{1}(\epsilon)}{\left\langle\psi_{1}^{\dagger} P_{1} \psi_{1}\right\rangle_{\epsilon}}
$$

Recent developments have been made in stochastic transport codes in order to obtain adjoint flux [5]. In a stochastic approach one would evaluate the above expressions based on sampled events. Therefore the related estimators will not exhibits the distributions that are used in the events sampling. For instance using a collision $k$ at $\epsilon_{k}$ for a reaction $r$ emitting a neutron in $\epsilon_{k}^{\prime}$ the estimator $\tilde{S}_{\sigma_{r, i}\left(E_{k}\right)}^{\rho}$ of $S_{\sigma_{r, i}(E)}^{\rho}$ is computed as

$$
\tilde{S}_{\sigma_{r, i}\left(E_{k}\right)}^{\rho}=\sigma_{r, i}\left(E_{k}\right) \frac{\left[\tilde{v}_{r, i}\left(E_{k}\right) \psi_{1}^{\dagger}\left(\epsilon_{k}^{\prime}\right)-\psi_{1}^{\dagger}\left(\epsilon_{k}\right)\right] N_{i}\left(\vec{r}_{k}\right) \psi_{1}\left(\epsilon_{k}\right)}{\left\langle\psi_{1}^{\dagger} P_{1} \psi_{1}\right\rangle_{\epsilon}}
$$

where $\psi_{1}\left(\epsilon_{k}\right)$ is the collision-based flux estimator defined as

$$
\psi_{1}\left(\epsilon_{k}\right)=\frac{w_{k}}{\sum_{i} N_{i}\left(\vec{r}_{k}\right) \sigma_{\text {tot }, i}\left(E_{k}\right)}
$$

where $w_{k}$ is the statistical weight of the neutron just before the collision. Note that we always sample $\vec{r}_{k}{ }^{\prime}=\vec{r}_{k}$. The adjoint flux estimator $\psi_{1}^{\dagger}\left(\epsilon_{k}\right)$ can be obtained using the collision-based IFP method as [5]

$$
\psi_{k}^{\dagger}\left(\epsilon_{k}\right)=\prod_{i=1}^{L} \frac{k_{i}^{\text {step }}}{k_{\infty}}
$$

For the sensitivity to distribution, the estimator is

$$
\tilde{S}_{p_{r, i}\left(\epsilon_{k} \rightarrow \epsilon_{k}^{\prime}\right)}^{\rho}=\tilde{v}_{r, i}\left(E_{k}\right) \sigma_{r, i}\left(E_{k}\right) \frac{\psi_{1}^{\dagger}\left(\epsilon_{k}^{\prime}\right) N_{i}\left(\vec{r}_{k}\right) \psi_{1}\left(\epsilon_{k}\right)}{\left\langle\psi_{1}^{\dagger} P_{1} \psi_{1}\right\rangle_{\epsilon}}
$$

Here we used the IFP capability of the development version of the TRIPOLI4 code [6] in order to compute these sensitivity coefficients to nuclear data. TRIPOLI4 samples the events and compute the required adjoint flux estimators and records the results in binary files. These files are then read by an external utility program to compute the sensitivity scores.

\section{Verification with Perturbations}

The computation of sensitivity coefficients to cross sections where already well tested [6]. To verify our sensitivities to distributions, we used the same utility to compute perturbations. Indeed the perturbation formalism allows to compute exact perturbations, therefore the impact $\delta k_{\text {eff }}$ from a modification $\delta \chi_{i}\left(E \rightarrow E^{\prime}\right)$ or $\delta p_{\text {scat }, i}\left(\epsilon \rightarrow \epsilon^{\prime}\right)$ can be obtained with expressions close to Eq. 3. The reference state corresponds to the simulation using a set of nuclear data to compute the direct flux $\psi_{1}$ whereas for the perturbed state a different set of nuclear data yields the adjoint flux $\psi_{2}^{\dagger}$. Our developments have been tested on the computation of exact perturbations on nuclear data distributions. We modified the JEFF-3.1.1 ${ }^{239} \mathrm{Pu}$ evaluation and produce 4 sets of ${ }^{239} \mathrm{Pu}$ simplified libraries: for instance we took the lowest-energy elastic angular distribution and applied it to 
the whole energy range. This produce the library named $\mathrm{AD}^{\text {low }}$, additionally the angular distribution for the highest incident energy has been applied in the whole energy range yielding the library $\mathrm{AD}^{\text {high }}$. A similar procedure has been applied to the fission spectrum yielding libraries $\chi^{\text {low }}$ and $\chi^{\text {high }}$. In these latter libraries we removed the delayed neutrons to simplify the comparison. The two extreme distributions are shown in Fig. 1.

Cosine of scattering angle in $\mathrm{CM} \mu_{\mathrm{CM}}$

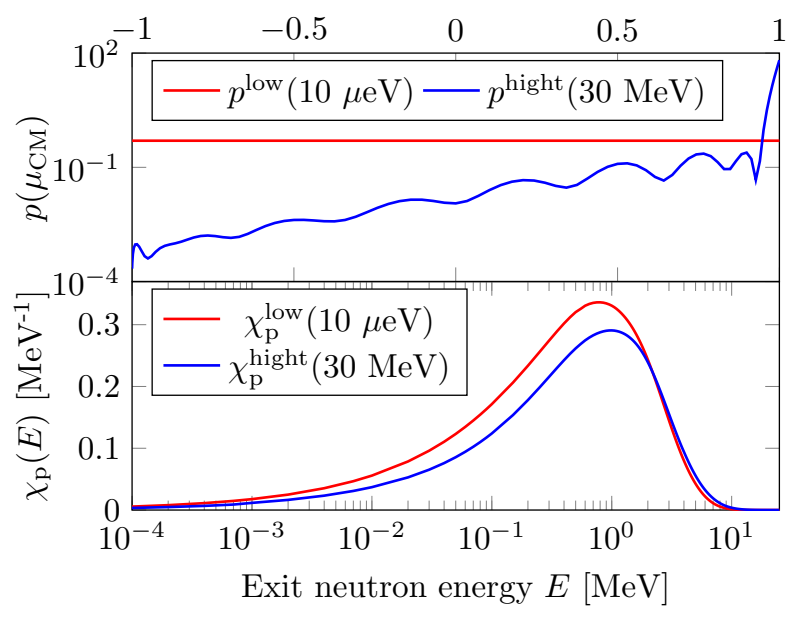

Figure 1. Two ${ }^{239} \mathrm{Pu}$ extreme elastic scattering angular distributions and fission spectra used to test the perturbation calculation.

We compute the $\rho$ change from a "low" library to the "high" one with direct calculations and with the perturba-

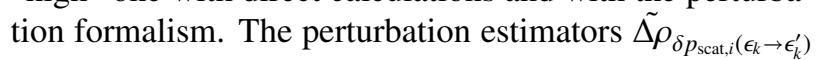
and $\tilde{\Delta \rho_{\chi_{i}\left(E_{k} \rightarrow E_{k}^{\prime}\right)}}$ for the scattering angular distribution and for the fission spectrum are respectively

$$
\begin{aligned}
& \tilde{\Delta}_{\delta p_{\text {scat }, i}\left(\epsilon_{k} \rightarrow \epsilon_{k}^{\prime}\right)} \\
& =\sigma_{\text {scat }, i}\left(E_{k}\right) \frac{\psi_{1}^{\dagger}\left(\epsilon_{k}^{\prime}\right) \frac{\delta p_{\text {satat } i}\left(\epsilon_{k} \rightarrow \epsilon_{k}^{\prime}\right)}{p_{\text {scat } i}\left(\epsilon_{k} \rightarrow \epsilon_{k}^{\prime}\right)} N_{i}\left(\vec{r}_{k}\right) \psi_{1}\left(\epsilon_{k}\right)}{\left\langle\psi_{1}^{\dagger} P_{1} \psi_{1}\right\rangle} \\
& \tilde{\Delta \rho_{\delta \chi_{i}\left(E_{k} \rightarrow E_{k}^{\prime}\right)}} \\
& =\frac{\bar{v}_{i}\left(E_{k}\right) \sigma_{\text {fiss }, i}\left(E_{k}\right)}{k_{\text {eff }}} \frac{\psi_{1}^{\dagger}\left(\epsilon_{k}^{\prime}\right) \frac{\delta \chi_{i}\left(E_{k} \rightarrow E_{k}^{\prime}\right)}{\chi_{i}^{\text {dit }}\left(E_{k} \rightarrow E_{k}^{\prime}\right)} N_{i}\left(\vec{r}_{k}\right) \psi_{1}\left(\epsilon_{k}\right)}{\left\langle\psi_{1}^{\dagger} P_{1} \psi_{1}\right\rangle}
\end{aligned}
$$

This test was performed on a simplify version of the ICSBEP benchmark PMF001 [7] containing only ${ }^{239} \mathrm{Pu}$, the corresponding results are shown in Tab. 1. The results are in good agreement thus validating the computation of the sensitivity coefficients to distributions.

Table 1. Perturbation results on PMF001, values are in $\mathrm{pcm}$.

\begin{tabular}{ccc}
\hline Library $\psi_{1} \rightarrow \psi_{2}^{\dagger}$ & Direct & Perturbation \\
\hline $\mathrm{AD}^{\text {low }} \rightarrow \mathrm{AD}^{\text {high }}$ & $1823 \pm 3$ & $1793 \pm 15$ \\
$\chi^{\text {low }} \rightarrow \chi^{\text {high }}$ & $-16305 \pm 4$ & $-16057 \pm 573$ \\
\hline
\end{tabular}

\section{Application to Resonance Parameters}

To compute sensitivities to resonance parameters the above sensitivities to cross sections and distributions must be "convoluted" with sensitivities to resonance parameters. Namely the sensitivity of $k_{\text {eff }}$ to a given resonance parameter $q$ reads

$$
\begin{aligned}
S_{q}^{\rho}=q\left[\sum_{r} \int \mathrm{d} E \frac{\partial \rho}{\partial \sigma_{r}(E)} \frac{\partial \sigma_{r}(E)}{\partial q}\right. \\
\left.\quad+\int \mathrm{d} \epsilon \int \mathrm{d} \epsilon^{\prime} \frac{\partial \rho}{\partial p_{\text {scat }}\left(\epsilon \rightarrow \epsilon^{\prime}\right)} \frac{\partial p_{\text {scat }}\left(\epsilon \rightarrow \epsilon^{\prime}\right)}{\partial q}\right]
\end{aligned}
$$

We omitted the index $i$ in the cross sections and distribution as a resonance parameters impacts only a single isotope. We restrict sum over distributions to the scattering one, therefore

$$
p_{\mathrm{scat}}\left(\epsilon \rightarrow \epsilon^{\prime}\right)=\frac{p_{\mathrm{el}}(E, \mu)}{2 \pi} \delta_{\vec{r}, \vec{r}^{\prime}} \delta_{\hat{\Omega} \cdot \hat{\Omega}^{\prime}, \mu}
$$

and

$$
p_{\mathrm{el}}(E, \mu)=\frac{2 \pi}{\sigma_{\mathrm{el}}(E)} \frac{\partial^{2} \sigma_{\mathrm{el}}(E, \mu)}{\partial \hat{\Omega}^{2}}
$$

where

$$
\frac{\partial^{2} \sigma_{\mathrm{el}}(E, \mu)}{\partial \hat{\Omega}^{2}}=\sum_{l=0}^{\ell} a_{l}(E) P_{l}(\mu) \text { and } a_{0}(E)=\frac{\sigma_{\mathrm{el}}(E)}{4 \pi}
$$

Using the sensitivity coefficients computed with the perturbation theory we obtain

$$
\begin{aligned}
S_{q}^{\rho}=\left[\sum_{r} \int \mathrm{d} E \frac{q}{\sigma_{r}(E)} \frac{\partial \sigma_{r}(E)}{\partial q} S_{\sigma_{r}(E)}^{\rho}\right. \\
\left.+\int \mathrm{d} \epsilon \int \mathrm{d} \epsilon^{\prime} S_{p_{r, i}\left(\epsilon \rightarrow \epsilon^{\prime}\right)}^{k_{\mathrm{ef}}} \frac{q}{p_{\mathrm{el}}(E, \mu)} \frac{\partial p_{\mathrm{el}}(E, \mu)}{\partial q}\right]
\end{aligned}
$$

So that

$$
\frac{\partial p_{\mathrm{el}}(E, \mu)}{\partial q}=\sum_{l=0}^{\ell}\left(\frac{\partial a_{l}(E)}{\partial q}-\frac{a_{l}(E)}{a_{0}(E)} \frac{\partial a_{0}(E)}{\partial q}\right) \frac{P_{l}(\mu)}{2 a_{0}(E)}
$$

We coupled the utility program with the CONRAD code [8] to compute the derivatives $\partial \sigma_{r} / \partial q$ and $\partial a_{l} / \partial q$. The CONRAD code was also used to produce the ENDF files that have been used by TRIPOLI4 so that consistency is ensured in our comparisons. The computed ${ }^{239} \mathrm{Pu}$ sensitivities to cross sections are shown in Fig. 2 as well as the resonance parameter sensitivities in the case of the PST001 benchmark. One can see that the resonance parameters related to the first positive resonance are the most sensitive parameters. The computed sensitivities to the first resonance of ${ }^{239} \mathrm{Pu}$ are given in Tab. 2 and compared with the results obtained with direct calculations by taking a variation of $\pm 1 \%$ of the parameter value.

In the JEFF-3.1.1 evaluation all ${ }^{239} \mathrm{Pu}$ resonance have an orbital momentum $\ell=0$ so that the angular distribution of the scattered neutrons is isotropic regardless of the resonance parameters. To see the impact of the inclusion of the angular distribution in the computation of $S_{q}^{k_{\text {eff }}}$ we 


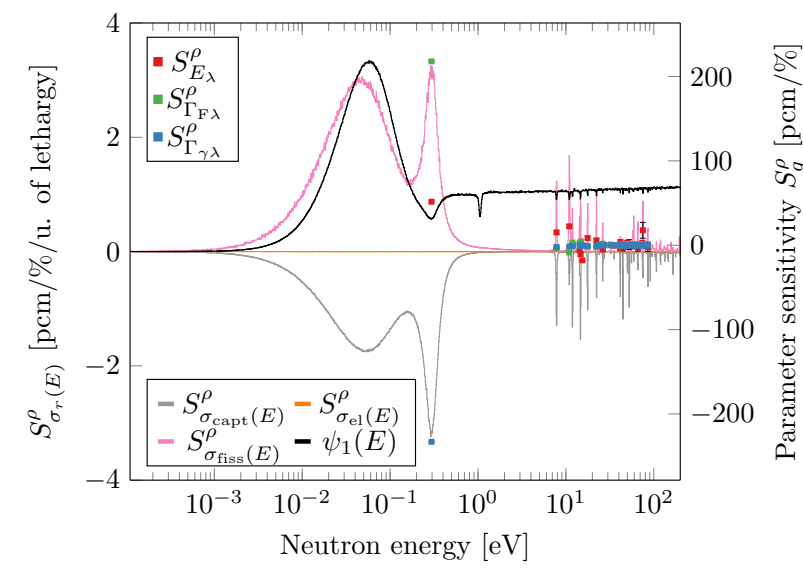

Figure 2. ${ }^{239} \mathrm{Pu}$ cross section and resonance parameter sensitivities for the PST001 benchmark. The neutron flux is also shown in arbitrary unit.

Table 2. Sensitivities to the first ${ }^{239} \mathrm{Pu}$ resonance parameters for the PST001 benchmark

\begin{tabular}{crr}
\hline \multicolumn{3}{c}{ Sensitivity $S_{q}^{\rho}[\mathbf{p c m} / \%]$} \\
Parameter $q$ & \multicolumn{1}{c}{ Direct } & \multicolumn{1}{c}{ IFP } \\
\hline$E_{1}$ & $51.8 \pm 0.3$ & $52.8 \pm 1.4$ \\
$\Gamma_{\mathrm{N} 1}$ & $-19.1 \pm 0.3$ & $-19.3 \pm 0.3$ \\
$\Gamma_{\gamma 1}$ & $-232.6 \pm 0.3$ & $-233.4 \pm 0.4$ \\
$\Gamma_{\mathrm{F} 1}$ & $216.5 \pm 0.3$ & $217.5 \pm 0.3$ \\
\hline
\end{tabular}

considered ${ }^{16} \mathrm{O}$ that has resonances at high energies with higher orbital momentum. The results for this isotope is show in Fig. 3, where we added the sensitivity to the distribution summed over initial directions $\hat{\Omega}$ and position $\vec{r}$ and final states $\epsilon^{\prime}$, namely

$$
S_{\sigma_{\mathrm{ad}}(E)}^{\rho}=\int \mathrm{d}^{2} \hat{\Omega} \int \mathrm{d}^{3} \vec{r} \int \mathrm{d} \epsilon^{\prime} S_{p_{\mathrm{scat}, i}\left(\epsilon \rightarrow \epsilon^{\prime}\right)}^{\rho}
$$

We focused on the second resonance near $1 \mathrm{MeV}$ with

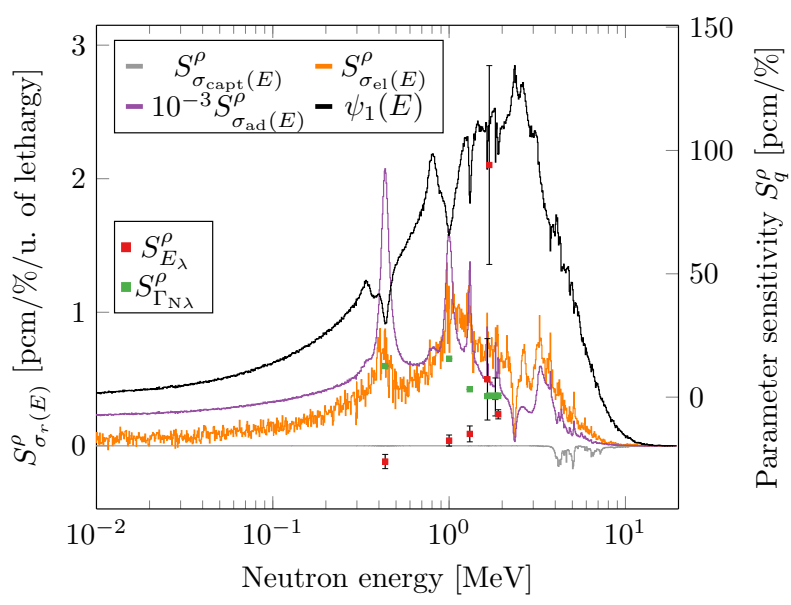

Figure 3. ${ }^{16} \mathrm{O}$ cross section, distribution and resonance parameter sensitivities for the PST001 benchmark. The neutron flux is also shown in arbitrary unit. $\ell=2$ that has the strongest sensitivity to parameter $\Gamma_{\mathrm{N}}$. We split the results in Tab. 3 with (AD) and without (NAD) inclusion of the angular distribution component. Comparison are also made with direct calculations taking again $\pm 1 \%$ of the parameters value. One should note that the angular distribution increases the total sensitivity by about $50 \%$. The small differences in the case of ${ }^{16} \mathrm{O}$ are believed to be due to a too small IFP cycle length (here 20).

Table 3. Sensitivity to the second ${ }^{16} \mathrm{O}$ resonance parameters on the PST001 benchmark

\begin{tabular}{cccc}
\hline \multicolumn{4}{c}{ Sensitivity $S_{q}^{\rho}[\mathrm{pcm} / \%]$} \\
Parameter $q$ & Direct & IFP \\
\hline$E_{2}$ & $\mathrm{AD}$ & $-15.9 \pm 0.2$ & $-18.7 \pm 2.6$ \\
$E_{2} \mathrm{NAD}$ & $-11.1 \pm 0.3$ & $-15.1 \pm 2.3$ \\
\hline$\Gamma_{\mathrm{N} 2}$ & $\mathrm{AD}$ & $11.5 \pm 0.2$ & $12.2 \pm 0.7$ \\
$\Gamma_{\mathrm{N} 2} \mathrm{NAD}$ & $10.9 \pm 0.3$ & $11.7 \pm 0.1$ \\
\hline
\end{tabular}

\section{Conclusion}

In this work we have used the collision-based IFP method to compute exact perturbations to distribution as well as reference sensitivity coefficients to evaluated resonance parameters. This sensitivities takes into account both impact of the resonance parameters to the cross sections and scattering distributions. We showed on an ICSBEP benchmarks that this latter component can amount up to a third of the total sensitivity. In future this methods will be applied to higher-energy nuclear models used to produce evaluations, namely optical model parameters.

\section{References}

[1] E. Brun, F. Damian, C. Diop, E. Dumonteil, F. Hugot, C. Jouanne, Y. Lee, F. Malvagi, A. Mazzolo, O. Petit et al., Annals of Nuclear Energy 82, 151 (2015), joint International Conference on Supercomputing in Nuclear Applications and Monte Carlo 2013

[2] A.M. Lane, R.G. Thomas, Rev. Mod. Phys. 30, 257 (1958)

[3] M. Aufiero, A. Bidaud, M. Fratoni, pp. 826-830 (2016)

[4] M. Williams, Perturbation theory for nuclear reactor analysis (1986), Vol. 3, pp. 63-188

[5] Y. Nauchi, T. Kameyama, Journal of Nuclear Science and Technology pp. 977-990 (2010)

[6] G. Truchet, P. Leconte, A. Santamarina, E. Brun, F. Damian, A. Zoia, Annals of Nuclear Energy 85, 17 (2015)

[7] OECD-NEA, International Handbook of Evaluated Criticality Safety Benchmark Experiments (Springer, 2018)

[8] P. Archier, C. De Saint Jean, O. Litaize, G. Noguère, L. Berge, E. Privas, P. Tamagno, Nuclear Data Sheets 118, 488 (2014) 